\title{
Variabilidade entre Cardiologistas na Abordagem aos Pacientes em Prevenção Secundária da Cardiopatia Isquêmica
}

\author{
Ricardo Stein, Caroline Alboim, Candice Campos, Renato Bandeira de Mello, \\ Guido Aranha Rosito, Carisi Anne Polanczyk \\ Porto Alegre, RS
}

\section{Objetivo}

Comparar o manejo dos pacientes com cardiopatia isquêmica, atendidos por cardiologistas em ambulatório geral de cardiologia, com aquele realizado em ambulatório específico de cardiopatia isquêmica, dando ênfase à solicitação do perfil lipídico e ao tratamento farmacológico prescrito.

\section{Métodos}

Dados obtidos de prontuário médico de 52 pacientes consecutivos atendidos no ambulatório de cardiopatia isquêmica (grupo I) e de 43 acompanhados no ambulatório de cardiologia geral (grupo II), sendo o diagnóstico anatômico de cardiopatia isquêmica, condição básica para sua inclusão. Considerou-se critério para dislipidemia, colesterol total $\geq 200 \mathrm{mg} / \mathrm{dl}$ e/ou LDL colesterol > $100 \mathrm{mg} / \mathrm{dl}$ em pacientes em uso ou não de hipolipemiantes e o uso de drogas hipolipemiantes, mesmo quando os níveis de colesterol total e/ou LDL colesterol eram < a 200 $\mathrm{mg} / \mathrm{dl}$ e $100 \mathrm{mg} / \mathrm{dl}$, respectivamente. Usou-se teste exato de Fischer para comparação de variáveis, aceitando como significativo $p$ bicaudal $<0,05$.

\section{Resultados}

Características demográficas, fatores de risco observados para cardiopatia isquêmica, prevalência de infarto do miocárdio prévio e procedimentos de revascularização prévios não apresentaram diferenças significativas entre os pacientes do grupo I e II. Observou-se que os pacientes do grupo I receberam aspirina em $98 \%$ dos casos, enquanto que no grupo II, sua prescrição foi de $83 \%(p=0,02)$. Em relação a drogas hipolipemiantes, as prevalências foram de $60 \%$ no grupo I e de $19 \%$ no grupo II $(p=0,001)$. Exames de perfil lipídico foram solicitados para $98 \%$ dos indivíduos do grupo / e $79 \%$ do grupo // $(p=0,003)$.

\section{Conclusão}

Houve maior fidedignidade de condutas no ambulatório específico de cardiopatia isquêmica em relação às novas evidências médicas, principalmente no que tange à prescrição de aspirina e de drogas hipolipemiantes.

\section{Palavras chaves \\ cardiopatia isquêmica, prevenção secundária, coorte ambulatorial}

Hospital de Clínicas de Porto Alegre - Ambulatório Especializado em Cardiopatia Isquêmica

Endereço para Correspondência: Dr. Ricardo Stein - R. João Caetano, 20/302 - Porto Alegre, RS - Cep 90470-260

E-mail: kuqui2@terra.com.br

Recebido para publicação em 5/9/03

Aceito em 4/2/04
$\mathrm{O}$ alto risco de morbimortalidade subseqüente à manifestação da cardiopatia isquêmica torna fundamental a instituição de regimes preventivos efetivos como parte do manejo geral de pacientes coronariopatas. Cerca de $8 \%$ dos sobreviventes de infarto agudo do miocárdio morrem ao longo do $1^{\circ}$ ano de seguimento. A partir do $2^{\circ}$ ano após evento agudo, a mortalidade varia entre 2 e $4 \%$ ao ano ${ }^{1}$. Cabe salientar que proporção adicional de pacientes apresenta como primeira manifestação novo infarto ou morte, sendo assim justificado o emprego de medicamentos ou de intervenções não farmacológicas, inclusive para os portadores de doença assintomática ${ }^{2}$. Essas medidas de prevenção secundária incluem controle dos fatores de risco coronarianos, exercício físico, uso de medicamentos, como betabloqueadores, inibidores da ECA, hipolipemiantes e anti-agregantes plaquetários e ainda, quando indicados, procedimentos de revascularização miocárdica ${ }^{3}$.

Apesar das evidências do benefício clínico dessas intervenções farmacológicas, são observadas variações importantes na abordagem terapêutica prescrita na doença aterosclerótica. Alguns fármacos que se encontram formalmente indicados para o tratamento da coronariopatia parecem ser subutilizados. Além disso, em outras circunstâncias, quando estes fármacos são prescritos, as doses utilizadas são menores do que as testadas e comprovadas em ensaios clínicos. Logo, parece clara a existência de uma lacuna entre o tratamento baseado nas melhores evidências científicas, com eficácia comprovada em ensaios clínicos, e a prática clínica usual $^{4}$. Estudos recentes conduzidos em outros países apontaram diferenças no manejo da cardiopatia isquêmica entre médicos cardiologistas e generalistas, com impacto inclusive na morbimortalidade desses pacientes ${ }^{5-7}$. As diferenças descritas entre especialistas podem ser resultantes da formação médica, infra-estrutura e motivadores para prática médica ou fatores culturais, não ficando claro qual fator é predominante e se esses achados podem ser extrapoláveis para o nosso meio.

Foi nosso objetivo comparar o manejo dos pacientes com cardiopatia isquêmica atendidos por cardiologistas em ambulatório geral de cardiologia, com aquele realizado em ambulatório especializado em cardiopatia isquêmica, verificando a qualidade do atendimento em ambos modelos assistenciais aplicados dentro de um centro de referência terciário de Porto Alegre.

\section{Métodos}

Entre os meses de abril e setembro de 2002, foram atendidos de forma consecutiva, 95 pacientes com diagnóstico de cardiopatia is- 
quêmica. Para fins desta investigação, considerou-se como cardiopata isquêmico o indivíduo com achados de cateterismo cardíaco evidenciando lesão aterosclerótica com estenose luminal $>50 \%$ em pelo menos uma artéria coronária (diagnóstico anatômico). Esses pacientes, dependendo do grupo a que pertenciam, foram atendidos em um de dois grupos ambulatoriais da cardiologia do Hospital de Clínicas de Porto Alegre (HCPA): ambulatório especializado em cardiopatia isquêmica e ambulatório geral de cardiologia.

Por meio de estudo transversal retrospectivo, foram coletados, de forma padronizada, dados de prontuários de pacientes. O HCPA é um hospital de atenção terciária, público, que atende predominantemente pacientes do Sistema Único de Saúde. Dentro de suas dependências, seis estudantes de medicina previamente treinados revisaram os prontuários e coletaram os dados das prescrições contidas na última consulta ambulatorial do paciente. Foram atendidos no ambulatório de cardiopatia isquêmica 52 indivíduos que constituíram o grupo I, enquanto outros 43 foram atendidos no ambulatório de cardiologia geral, formando o grupo II. No ambulatório de cardiopatia isquêmica, os pacientes eram atendidos por alunos de graduação e pós-graduação da Universidade Federal do Rio Grande do Sul (UFRGS), com orientação de mestres e doutores cardiologistas. No ambulatório de Cardiologia Geral, o atendimento era prestado por médicos residentes sob a supervisão de médicos cardiologistas e professores do HCPA.

Coletaram-se dados demográficos, história clínica, exames laboratoriais e procedimentos prévios, com ênfase na solicitação de perfil lipídico e no tratamento farmacológico prescrito. No presente estudo, considerou-se critério para dislipidemia, colesterol total $\geq 200 \mathrm{mg} / \mathrm{dl}$ e/ou LDL colesterol > $100 \mathrm{mg} / \mathrm{dl}$ em pacientes em uso ou não de hipolipemiantes. Também foi utilizado como critério para dislipidemia o uso de drogas hipolipemiantes, mesmo quando os níveis de colesterol total e/ou LDL colesterol eram $<$ a $200 \mathrm{mg} / \mathrm{dl}$ e $100 \mathrm{mg} / \mathrm{dl}$, respectivamente.

Os dados de ambos grupos foram comparados, sendo utilizado o teste exato de Fischer para determinar diferenças estatísticas entre eles. Considerou-se significativo o valor de $p$ bicaudal $<0,05$.

\section{Resultados}

As características demográficas, os fatores de risco observados para cardiopatia isquêmica, prevalência de infarto do miocárdio prévio e procedimentos de revascularização prévios não apresentaram diferenças significativas entre os pacientes atendidos, tanto no ambulatório específico de cardiopatia isquêmica, quanto no ambulatório de cardiologia geral (tab. I). Cabe salientar, especificamente, que não houve diferença significativa entre os dois grupos em relação a dados de perfil lipídico (tab. II).

A prescrição medicamentosa dos dois grupos foi comparada e não houve diferença significativa quanto ao uso de betabloqueadores, inibidores da ECA, nitratos e antagonistas do cálcio. Observou-se que os pacientes atendidos no ambulatório especializado de cardiopatia isquêmica receberam mais aspirina em comparação com aqueles atendidos no ambulatório de cardiologia geral (tab. III).

Durante o estudo, a solicitação de perfil lipídico foi mais freqüentemente avaliada nos pacientes do grupo I do que nos do grupo II. Entre os pacientes com dislipidemia $(n=51)$, houve maior prescrição de estatinas no grupo I em comparação ao grupo II

\begin{tabular}{|lccc|}
\hline \multicolumn{3}{|c|}{$\begin{array}{c}\text { Tabela I - Características demográficas e fatores de risco para doença } \\
\text { arterial coronária nos grupos avaliados }\end{array}$} \\
\hline & $\begin{array}{c}\text { Grupo I } \\
\mathrm{n}=52\end{array}$ & $\begin{array}{c}\text { Grupo II } \\
\mathrm{n}=43\end{array}$ & $\mathrm{p}$ \\
\hline & 62 & 62 & $\mathrm{NS}$ \\
Idade (anos) & 67 & 67 & $\mathrm{NS}$ \\
Sexo Masculino & 65 & 77 & NS \\
Hipertensão & 82 & 77 & NS \\
Diabetes Melittus & 65 & 39 & NS \\
Dislipidemia & 63 & 78 & NS \\
Tabagismo & 61 & 69 & NS \\
Infarto prévio & 31 & 21 & NS \\
ICP & 27 & 28 & NS \\
CRM & &
\end{tabular}

$\mathrm{ICP}=$ intervenção coronariana percutânea; $\mathrm{CRM}=$ cirurgia de revascularização miocárdica.

\begin{tabular}{|lccc|}
\hline \multicolumn{4}{|c|}{$\begin{array}{c}\text { Tabela II - Médias de perfil lipídico e porcentagem de indivíduos com } \\
\text { níveis ótimos de LDL-colesterol }\end{array}$} \\
\hline & $\begin{array}{c}\text { Grupo I } \\
\mathrm{n}=52 \text { (DP) }\end{array}$ & $\begin{array}{c}\text { Grupo II } \\
\mathrm{n}=43 \text { (DP) }\end{array}$ & $p$ \\
\hline Colesterol total & $216(24)$ & $226(28)$ & $\mathrm{NS}$ \\
LDL-colesterol & $142(12)$ & $152(15)$ & $\mathrm{NS}$ \\
HDL-colesterol & $38(6)$ & $37(5)$ & $\mathrm{NS}$ \\
Triglicerícios & $180(22)$ & $187(18)$ & $\mathrm{NS}$ \\
Níveis adequados de & 37 & 30 & $\mathrm{NS}$ \\
LDL-colesterol (\%) & & & \\
\hline DP = desvio padrão; Colesterol total; LDL e HDL expressos em mg/dl. \\
\hline
\end{tabular}

\begin{tabular}{|c|c|c|c|}
\hline \multicolumn{4}{|c|}{$\begin{array}{l}\text { Tabela III - Diferenças nas prescrições dos fármacos utilizados em } \\
\text { cardiopatia isquêmica }\end{array}$} \\
\hline & $\begin{array}{c}\text { Grupo I } \\
\mathrm{n}=52(\%)\end{array}$ & $\begin{array}{c}\text { Grupo II } \\
n=43(\%)\end{array}$ & Valor $p$ \\
\hline Beta-bloqueador & 58 & 51 & NS \\
\hline I-ECA & 40 & 39 & NS \\
\hline Nitrato & 52 & 35 & NS \\
\hline Antagonistas do Cálcio & 50 & 26 & NS \\
\hline Estatina & 60 & 19 & 0,001 \\
\hline Aspirina & 98 & 84 & 0,02 \\
\hline
\end{tabular}

\section{Discussão}

Nos últimos anos, grande ênfase foi dada para identificação de tratamentos que produzissem impacto sobre a morbimortalidade na cardiopatia isquêmica. Entretanto, as evidências científicas não são adotadas como prática clínica em muitas instituições. Neste estudo retrospectivo de delineamento transversal foram demonstradas algumas diferenças, tanto no controle dos fatores de risco de cardiopatia isquêmica quanto no tratamento propriamente dito nos ambulatórios estudados. O perfil lipídico foi mais solicitado no ambulatório específico de cardiopatia isquêmica e, conseqüentemente, o uso de hipolipemiante nesse ambulatório foi significativamente maior. Embora a aspirina tenha sido significativamente mais prescrita neste grupo, não houve diferença estatística quanto ao uso de betabloqueadores, inibidores da ECA, nitratos e antagonistas do cálcio.

A prevalência das características demográficas e dos fatores de risco para doença arterial coronariana encontrada (tab. I) assemelha-se àquela observada na população em geral, fato este evidenciado pelos dados publicados no EUROASPIRE I e $\|^{8,9}$.

O EUROASPIRE II foi um estudo conduzido em 15 países da 
Europa e teve como objetivo principal avaliar se as recomendações estabelecidas para prevenção secundária de coronariopatia no EUROASPIRE I estavam sendo seguidas na prática clínica, alguns anos após. Nossos dados se assemelham aos dados europeus, uma vez que o uso de estatinas no EUROASPIRE II foi de $61 \%$ e no grupo I de nossa amostra foi de $60 \%$. No estudo multicêntrico europeu, o uso da aspirina também foi semelhante ao do grupo I do nosso estudo observacional, sendo $86 \%$ no EUROASPIRE II e de $98 \%$ no grupo específico de cardiopatia isquêmica (84\% no grupo II).

0 uso da aspirina está associado à redução de mortalidade em 17 a 30\%, na prevenção secundária. Reduz em aproximadamente $50 \%$ a oclusão de pontes após cirurgia de revascularização miocárdica, além de diminuir as complicações oclusivas agudas após angioplastia coronariana transluminal percutânea (ACTP) ${ }^{10,11}$. Em associação ao supracitado, a aspirina é uma droga barata e de fácil administração (tomada única diária). Pelos motivos expostos, a adequação de sua indicação em prevenção secundária é fundamental, refletindo na alta prevalência de seu uso, especialmente em ambulatório especializado.

Estudos epidemiológicos apontam para uma associação positiva entre níveis elevados de colesterol sérico e a incidência de doença aterosclerótica. Sabe-se que há uma redução significativa na morbimortalidade da doença coronariana com o uso de hipolipemiantes, principalmente inibidores HMG-CoA (estatinas). É importante acrescentar o benefício com o uso das estatinas mesmo naqueles indivíduos com níveis normais e/ou baixos de LDL-C, desde que sejam classificados como de alto risco ${ }^{12-14}$. Em prevenção secundária, a utilização de estatinas apresenta uma diminuição de $42 \%$ no risco de mortalidade cardiovascular, com benefício absoluto após seis anos de 4 vidas salvas, 7 infartos prevenidos e 6 cirurgias de revascularização miocárdica evitadas em cada 100 pacientes tra$\operatorname{tados}^{15}$. Nesse grande ensaio clínico, o número de pacientes tratados necessários para salvar uma vida (NNT) foi de 25. Além disso, assim como a aspirina, as estatinas também são administradas em tomada única, facilitando sua aderência, porém seu custo elevado pode ser um fator limitante à adesão.

Acredita-se que os resultados clínicos relacionados a condutas efetuadas por cardiologistas possam ser mais efetivos do que aqueles provenientes de condutas prestadas por médicos internistas, especificamente em se tratando do manejo de pacientes com doenças cardiológicas, entre elas a cardiopatia isquêmica. Dentro dessa linha de hipóteses, sugerimos que a terapêutica oferecida por subespecialistas em cardiopatia isquêmica seja superior à dos cardiologistas gerais. Em nosso entender, talvez possa ser devida ao conhecimento mais aprofundado na área em questão ou a um seguimento maior das orientações provenientes de diretrizes. Estudo realizado na Filadélfia comparou as condutas por médicos de família, internistas, cardiologistas autodesignados e cardiologistas com título de especialista no tratamento do infarto agudo do miocárdio ${ }^{5}$. Os resultados demonstraram que menor mortalidade foi associada ao tratamento por cardiologistas que haviam tratado maior número de pacientes com infarto agudo do miocárdio, estavam mais próximos (em anos) da graduação da faculdade de medicina e apresentavam título de especialista. Outro estudo realizado em Boston, focado na diferença do manejo da prevenção de doenças cardiovasculares, mostrou que cardiologistas têm maior propensão a seguir estratégias de prevenção primária, como testes de colesterol, prescrição de dieta e de exercício físico, além da mensuração do peso corporal. Por outro lado, os médicos generalistas variam amplamente em suas condutas quanto à prevenção primária ${ }^{6}$. Em outro estudo de prevenção secundária realizado na Califórnia, uma população de pacientes dislipidêmicos pós-infarto recebeu duas vezes mais prescrição de hipolipemiantes, quando atendidos por cardiologistas, que seus pares atendidos por internistas ${ }^{7}$. Nesta mesma linha de observação, estudo publicado no final dos anos noventa explicita que os betabloqueadores são subutilizados em tratamento ambulatorial de pacientes com cardiopatia isquêmica, principalmente entre médicos de família e internistas ${ }^{16}$.

Nossos dados apontam maior eficácia no atendimento aos pacientes coronariopatas no ambulatório de cardiopatia isquêmica em relação àqueles atendidos no ambulatório de cardiologia geral, no sentido de atingir melhor controle de fatores de risco, demonstrado pela tendência verificada na tabela III (diferença na prescrição de fármacos que modificam a história natural da doença arterial coronariana. No entanto, este estudo não é isento de limitações. Por apresentar delineamento transversal prospectivo, a avaliação de adequação do controle de fatores de risco não pode ser o foco deste experimento. Indo ao encontro do que já foi citado, salientamos que o tempo médio de seguimento em nossa amostra, ao longo de todo o período deste estudo, foi muito curto, o que também não permite uma avaliação apropriada de efetividade no controle de fatores de risco.

Dados recentemente apresentados pelo nosso grupo ${ }^{17}$, tendo como base de investigação uma coorte ambulatorial robusta, demonstraram melhora significativa no controle de fatores de risco entre os indivíduos acompanhados no ambulatório especializado em cardiopatia isquêmica ao longo de um período de 21 meses.

Esclarecemos, também, que podem ter ocorrido diferenças de qualidade no registro de dados de pacientes atendidos no ambulatório de cardiologia geral, uma vez que nesses locais não há o seguimento de protocolos específicos para coleta e armazenamento de dados, o que pode provocar viéses de aferição, tanto em uma direção quanto em outra.

Concluindo, evidências observacionais de outros países sugerem que o tratamento é tanto mais adequado quanto mais o médico assistente for especializado na área. Nosso estudo reforça a hipótese de que o acompanhamento dos pacientes com cardiopatia isquêmica por cardiologistas especialistas nesta síndrome seja um elemento de impacto terapêutico e de efetividade na implementação de diretrizes embasadas nas melhores evidências disponíveis.

Estratégias de ampliação e aplicabilidade destas diretrizes passam por ambulatórios especializados com equipes multidisciplinares, programas de treinamento de generalistas e acesso facilitados à consultoria. Portanto, nossos dados sugerem que o atendimento em ambulatório especializado em cardiopatia isquêmica seja mais eficaz do que aquele prestado pelo cardiologista geral que atende o cardiopata isquêmico. 


\section{Referências}

1. Launberg J, Fruergaard P et al. Ten year mortality in patients with suspected acute myocardial infarction. BMJ 1994; 308: 1196-9.

2. Aronow WS. Silent MI. Prevalence and prognosis in older patients diagnosed by routine electrocardiograms. Geriatrics 2003; 58: 24-6, 36-8, 40.

3. Raymond J. Gibbons, Jonathan Abrams, Kanu Chatteriee et al. ACC/AHA 2002 guideline update for the management of patients with chronic stable angina-summary article [ACC/AHA guideline]. J Am Coll Cardiol, 2003, 41, Issue1.

4. McAlister FA, Taylor Gibbons RJ, Abrams J, Chatteriec KL et al. The treatment and prevention of coronary heart disease in Canada: do older patients receive efficacious therapies? The Clinical Quality Improvement Network (CQIN) Investigators. J Am Geriatr Soc. 1999; 47: 811-8.

5. Norcini JJ, Kinball HR, Lipner RS. Certification and specialization: do they matter in the outcome of acute myocardial infarction? Acad Med. 2000; 75: 1193-8.

6. Stafford RS, Blumenthal D. Specialty differences in cardiovascular disease prevention practices. J Am Coll Cardiol. 1998; 32: 1238-43.

7. Whyte JJ, Filly AL, Jollis JG. Treatment of hyperlipidemia by specialists versus generalists as secondary prevention of coronary artery disease. Am J Cardiol. 1997; 15, 80: 1345-7.

8. EUROASPIRE. A European Society of Cardiology survey of secondary prevention of coronary heart disease: principal results. EUROASPIRE Study Group. European Action on Secondary Prevention through Intervention to Reduce Events. Eur Heart J 1997; 18: 1569-82.

9. Lifestyle and risk factor management and use of drug therapies in coronary patients from 15 countries; principal results from EUROASPIRE II Euro Heart Survey
Programme. Eur Heart J 2001; 22: 554-72.

10. Collaborative overview of randomised trials of antiplatelet therapy-II: Maintenance of vascular graft or arterial patency by antiplatelet therapy. Antiplatelet Trialists' Collaboration. BMJ. 1994; 15; 308: 159-68.

11. Collaborative overview of randomised trials of antiplatelet therapy-I: Prevention of death, myocardial infarction, and stroke by prolonged antiplatelet therapy in various categories of patients. Antiplatelet Trialists' Collaboration. BMJ. 1994 308: 81-106.

12. The effect of pravastatin on coronary events after myocardial infarction in patients with average cholesterol levels. Cholesterol and Recurrent Events Trial investigators (CARE). N Engl J Med 1996; 335: 1001-9.

13. Prevention of cardiovascular events and death with pravastatin in patients with coronary heart disease and a broad range of initial cholesterol levels. The LongTerm Intervention with Pravastatin in Ischaemic Disease (LIPID) Study Group. N Engl J Med 1998; 339: 1349-57.

14. Heart Protection Study of cholesterol lowering with simvastatin in 20.536 high-risk individuals: a randomized placebo-controlled trial (HPS). Lancet 2002; 360: 7-22

15. The Scandinavian Simvastatin Survival Study Group. Randomized trial of cholesterol lowering in 4444 patients with coronary heart desease: the Scandinavian Simvastatin Survival Study (4S). Lancet 1994; 344: 1383-89

16. Wang TJ, Stafford RS. National patterns and predictors of beta-blocker use in patients with coronary artery disease. Arch Intern Med 1998 Sep 28; 158: 1901-6.

17. Mello RGB, Stein R, Polanczyk CA et al. Efetividade da terapia farmacológica baseada em evidência na prevenção secundária da cardiopatia isquêmica. Arq Bras Cardiol 2003, 81, (supl. III): 115. 